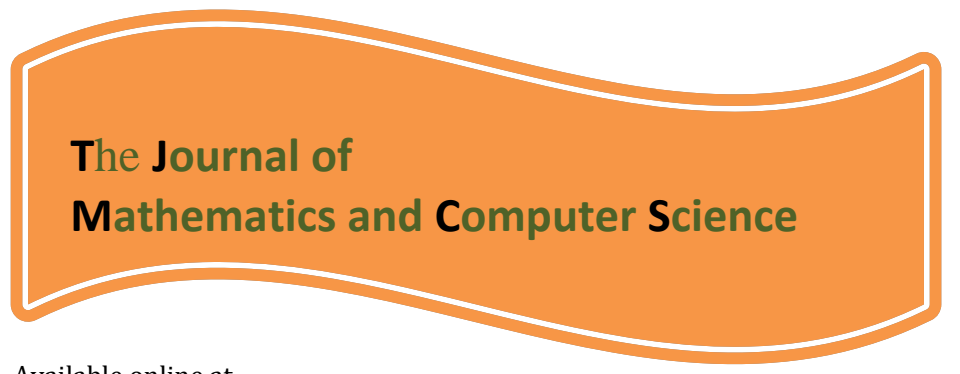

Available online at

http://www.TIMCS.com

The Journal of Mathematics and Computer Science Vol .2 No.2 (2011) 262-270

\title{
Evaluating the Reliability of Communication Networks (WAN) Using their Fuzzy Fault Tree Analysis - A Case Study
}

\author{
P. Rafiee ${ }^{1}$ and G. Latif Shabgahi ${ }^{2}$ \\ Computer Engineering, Network Expert of Keshavarzi Bank, Tehran,Iran, Email: p-rafiee@agri-bank.com \\ Control Dept,Faculty of Electrical Engineering, Power and Water University of Technology, Tehran, Iran
}

Received: September 2010, Revised: December 2010

Online Publication: January 2011

\begin{abstract}
Keeping wide area networks in a reliable mode, and evaluating their reliability is an essential task for network managers. Redundancy in components and communication links in networks is used to provide reliability. In this paper fault tree model is used to evaluate the reliability of an operational network. This analysis is a hierarchical and logical model of undesirable events of the system that is based on all possible combinations of its basic and intermediate events. Since there is often uncertainty in the estimation of failure probability of system components and communication links have been considered as a fuzzy system, and we have evaluated the system fault tree based on indefinite SNMP protocol by means of the fuzzy logic. We have described our methodology by means of a small case study and presented our results.
\end{abstract}

Keywords: Network Reliability, Fault Tree, Fuzzy Logic 


\section{Introduction}

Due to increasing use of communication networks and ease of communication in today's world , organizations, companies and particularly commercial sector would like to provide their services in geographically distributed location. Such a network provider link between headquarter and distributed branches enabling to use shared resources. In business and safety-critical system like ticket reservation and banking networks. the establishment of a stable and reliable connection between the system node is required. System designers employ redundancy methods to ensure sufficient reliability for their system the use of passive redundancy and spare standby arrangements in network components and communication links is a common practice.[1] In such arrangements, a link with more priority level is used as the primary data carrier, and in the case of occurring fault in primary carrier links, the links with the next priority level will be replaced by means of network protocol like HSRP [3]. Also, in some cases data will be communicated (as a Load Balance) through the two simultaneous communication links. In order to monitor and maintain the network in a reliable mode, assuring the reliability of network links is vital. In this paper, network reliability will be evaluated based on their fault tree model. Fault tree is a hierarchical and logical model of a system to find out the probability of its top event based on failure-rate probability of its basic and intermediate events. Considering network communications as a "system" and describe network disconnectivity as "undesirable top event", the aim of fault tree is to find out most critical points and faults in component and paths of the system for which the occurrence of fault has potential to disconnect expected services to system customer and user. In many cases. There is often uncertainty in the estimation of failure probability of network components and communication links, thus determining an acceptable value for distinguish the failure probability of top event is impossible. For example, setting a sharp boundary to "good" and "bad" bandwidth is unrealistic, because only a bit change in its LSB value may change "good BW" to "bad BW" and viceversa, fuzzy values should be used for failure probably of basic events. In other words, by using fuzzy inputs extracted from SNMP protocol the reliability of communication networks is assessed by means of fuzzy fault tree analysis. The organization of the paper is as follows. In the second section of this paper fuzzy logic will be discussed partially. And in the third section the system and input output and their fuzzy rules will be discussed, then by the fourth section after recognizing fault tree ,the network fault tree analysis will be done, and finally in the fifth section reliability o $\mathrm{f}$ communication networks will be evaluated by means of Fuzzy Fault Tree analysis.

\section{Fuzzy Logic}

Fuzzy logic is a powerful problem solving methodology with many applications in embedded control and information processing. Fuzzy provides a remarkably simple way to draw definite conclusions from vague, ambiguous or imprecise information. With fuzzy logic, propositions can be represented with degrees of "completely true" and "completely false". In a sense, fuzzy logic resembles human decision making with its ability to work from approximate data and find precise solutions. It can be implemented in hardware, software, or a combination of both.

\section{System Description}

In this paper Considering components and network communications as a system. System inputs will be obtained by using fuzzy inputs extracted from SNMP protocol and on the other hand system output represents status of network communication (Link State). Therefore, the system can be modeled as Figure (1)[5][6]. 


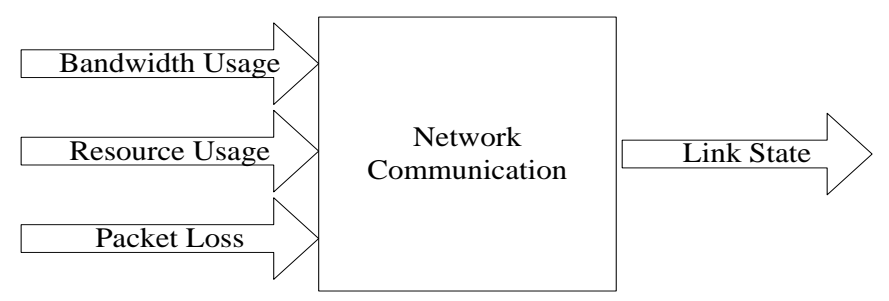

Figure (1).System description

Basic events are extracted from SNMP protocol, SNMP data cannot be used as definite inputs into network fault tree model because they have not a sharp boundary to describe link states. basic events are represented as fuzzy input. In the following sub setting, fuzzy inputs and outputs and system

rules are defined.

\section{3-1. Membership Functions of System Inputs}

The system has 3 inputs (bandwidth usage, packet lost and resource usage). In fact sufficient bandwidth is necessary for communication and data transmission. Percentage of bandwidth usage versus total bandwidth can be represented based on membership function of Figure (2). Using maximum bandwidth of link can increase the number of packets lost and makes a bottleneck at that point of network [7].

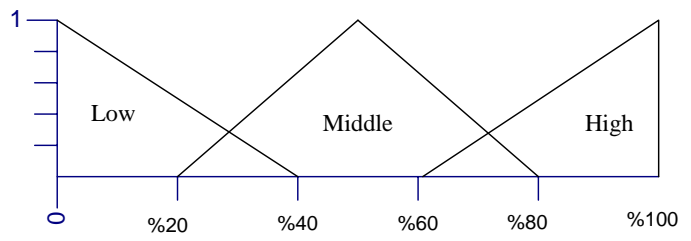

Figure (2).Bandwidth membership function

Packet lost is the other scale that occurs whenever noise happens in line or lack of bandwidth and can be formed with membership function Figure (3). Obviously, if the number of packet lost increase to hundred percent, it means that the network communication is disconnected [8].

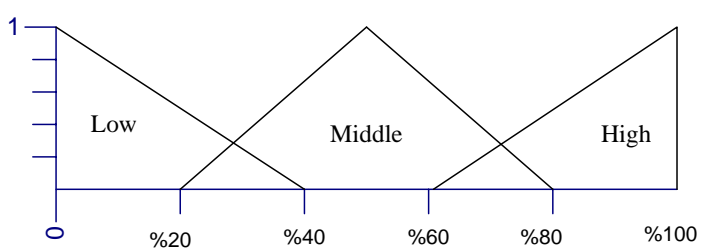

Figure (3). Packet lost membership function

Extra using of resources in single point of failures can make fault of components and make network disconnect, so the amount of CPU and RAM usage in switches and routers is important and will be obtained by the SNMP protocol. In this paper, resource usage can be represented with membership function Figure (4). 


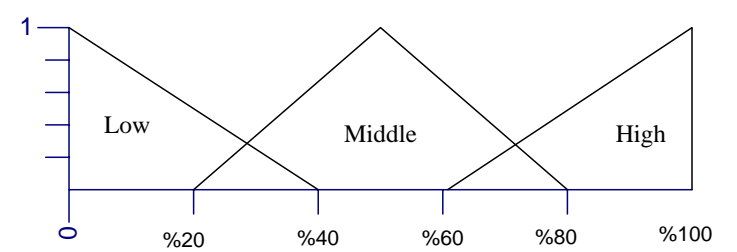

Figure (4). Resource usage membership function

Pay attention that more detailed measures such as RTD can be analyzed for more complex Network structure through the network protocols but for ease of expression we omitted those criteria.

\section{3-2. Membership Function of System Output}

Conditions of network communication can be presented based on the probability of failure of communication links and can be showed with membership functions Figure (5)

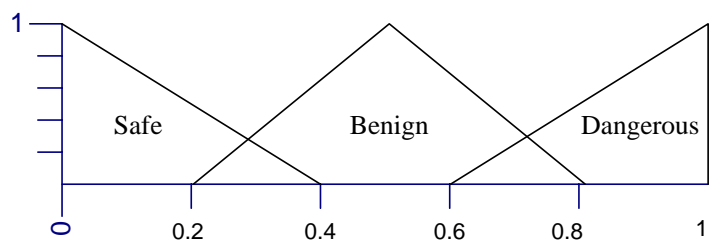

Figure (5). Output membership function (link State)

\section{3-3. Fuzzy Rules}

Rules associate ideas and relate one event to another. Fuzzy rules also operate using a series of ifthen statements, For instance, if X then A. Fuzzy rules are used to fuzzy logic mimics the crucial ability of the human mind to summarize data and focus on decision-relevant information. In fact, they represented relationship between input membership functions and output membership function. Fuzzy rules of described system have showed in the Table (1)[4].

\begin{tabular}{|c|c|c|c|c|c|c|c|c|c|c|c|c|c|c|c|c|c|c|c|c|c|c|c|c|c|c|c|c|}
\hline \multirow{3}{*}{ 营 } & $\begin{array}{c}\text { Bandwidth } \\
\text { Usage }\end{array}$ & 弪 & 3 & 茎 & $\begin{array}{l}3 \\
0 \\
-1\end{array}$ & $\begin{array}{l}3 \\
0 \\
-1\end{array}$ & 3 & $\begin{array}{l}3 \\
0 \\
\\
-1\end{array}$ & $\begin{array}{l}3 \\
0 \\
-1\end{array}$ & $\begin{array}{l}3 \\
0 \\
-1\end{array}$ & $\begin{array}{l}\frac{0}{\vec{\sigma}} \\
\stackrel{\vec{\nu}}{\Sigma}\end{array}$ & 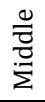 & $\begin{array}{l}\stackrel{0}{\vec{z}} \\
\stackrel{\vec{z}}{\Sigma}\end{array}$ & $\begin{array}{l}\frac{0}{\vec{z}} \\
\frac{\vec{z}}{\Sigma}\end{array}$ & 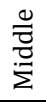 & 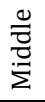 & $\begin{array}{l}\stackrel{0}{\vec{z}} \\
\stackrel{0}{\Sigma}\end{array}$ & $\frac{\varrho}{\bar{z}}$ & 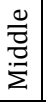 & 苟 & 㺃 & $\frac{}{500}$ & $\begin{array}{l}\frac{7}{600} \\
.7\end{array}$ & 苗 & 㺃 & $\begin{array}{l}7.5 \\
.00 \\
.1\end{array}$ & 垔 & $\frac{5}{600}$ \\
\hline & $\begin{array}{l}\text { Packet } \\
\text { Loss }\end{array}$ & 3 & 3 & 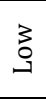 & $\begin{array}{l}\frac{0}{\tilde{z}} \\
\stackrel{0}{\tilde{\Sigma}} \\
\dot{\Sigma}\end{array}$ & $\begin{array}{l}\frac{0}{\tilde{z}} \\
\stackrel{0}{\Sigma} \\
\end{array}$ & 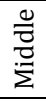 & 㿣 & 㺃 & 㺃 & 3 & 3 & $\begin{array}{l}3 \\
0 \\
0\end{array}$ & $\begin{array}{l}\frac{0}{\bar{z}} \\
\stackrel{\vec{z}}{\Sigma}\end{array}$ & $\begin{array}{l}\frac{0}{\tilde{\sigma}} \\
\stackrel{0}{\Sigma} \\
\end{array}$ & $\begin{array}{l}\frac{0}{\pi} \\
\frac{\pi}{2} \\
\end{array}$ & 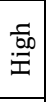 & 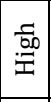 & 㺃 & 3 & 3 & $\begin{array}{l}3 \\
0 \\
9\end{array}$ & 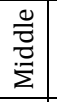 & $\begin{array}{l}\frac{0}{\tilde{z}} \\
\stackrel{0}{\tilde{\Sigma}} \\
\stackrel{\Sigma}{\Sigma}\end{array}$ & $\begin{array}{l}\frac{0}{\tilde{z}} \\
\frac{\pi}{\Sigma} \\
\end{array}$ & 釡 & 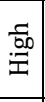 & $\frac{\sqrt{50}}{000}$ \\
\hline & $\begin{array}{c}\text { Resource } \\
\text { Usage }\end{array}$ & $\begin{array}{l}3 \\
0 \\
0 \\
9\end{array}$ & $\frac{\vec{\sigma}}{\bar{\sigma}}$ & $\begin{array}{l}5 \\
600 \\
017\end{array}$ & $\begin{array}{l}3 \\
0 \\
0\end{array}$ & 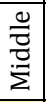 & 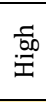 & $\begin{array}{l}3 \\
0 \\
9\end{array}$ & 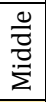 & 点 & 3 & 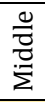 & $\begin{array}{l}\frac{5}{00} \\
00\end{array}$ & $\begin{array}{l}3 \\
0 \\
0\end{array}$ & 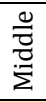 & 㺃 & $\begin{array}{l}3 \\
0 \\
9 \\
\end{array}$ & 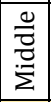 & 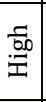 & 3. & 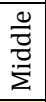 & $\begin{array}{l}\overrightarrow{5} \\
.00 \\
: 1\end{array}$ & 3 & 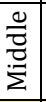 & 㺃 & $\begin{array}{l}3 \\
0 \\
0 \\
9\end{array}$ & 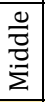 & $\begin{array}{l}\frac{1}{600} \\
: 10\end{array}$ \\
\hline 艺 & $\begin{array}{l}\text { Link } \\
\text { State }\end{array}$ & $\frac{\pi}{\pi}$ & $\stackrel{\Xi}{\sim}$ & 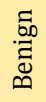 & 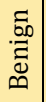 & 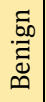 & 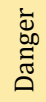 & 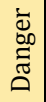 & 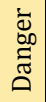 & 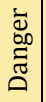 & $\underset{\pi}{\pi}$ & 䒕 &. & 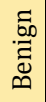 & 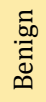 & 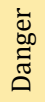 & 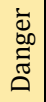 & 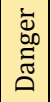 & 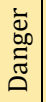 & 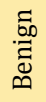 & 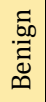 & 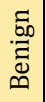 & 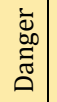 & 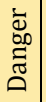 & 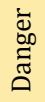 & 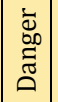 & 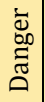 & $\begin{array}{l}\tilde{\Xi} \\
\infty \\
\tilde{\Xi} \\
\tilde{\Xi}\end{array}$ \\
\hline
\end{tabular}

\section{System Analysis}




\section{4-1.Fault Tree}

A system fault tree is a hierarchical and logical model of a system to find out the probability of its top event based on failure-rate probability of its basic and intermediate events. Also it is a graphical diagram that represented the top event in system based on and intermediate and basic events. Basic and intermediate events are related to each other with logic gates (AND, OR) and make the major system fault. Therefore, a fault tree can be a set of events and logic gates that forms an inverted tree which the major system fault is located on its top. Figure (5) shows a sample fault tree [2].

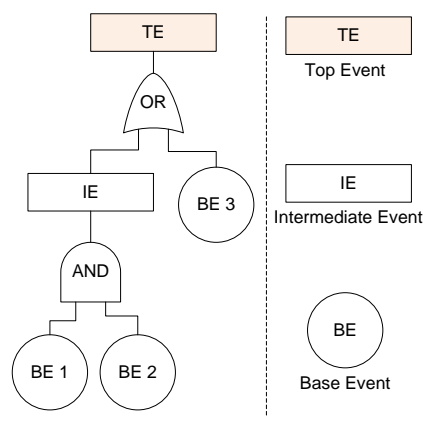

Figure (6).sample fault tree

To evaluate the probability of top event (vice versa system reliability), we must obtain probability of basic and intermediate events based on the logical relationships (AND/OR) between them and the tree structure, through the following two equations. Regarding to the following equations, top event probability of tree in Figure (6) can be obtained based on the inputs E1 and E2 [2].

$$
\begin{aligned}
& p(A N D)=p\left(E_{1}\right) \cdot p\left(E_{2}\right) \\
& p(O R) \cong p\left(E_{1}\right)+p\left(E_{2}\right)
\end{aligned}
$$

We evaluate probability of top event by using the above equations as following

$p(T E)=p\left(B E_{3}\right)+p\left(B E_{1}\right) \cdot p\left(B E_{2}\right)$

\section{4-2. Drawing the System Fault Tree}

As Figure (7) is observed, this network has a two-level hierarchical structure which communication between different levels with each other is established by parallel links and components (switches and routers). 


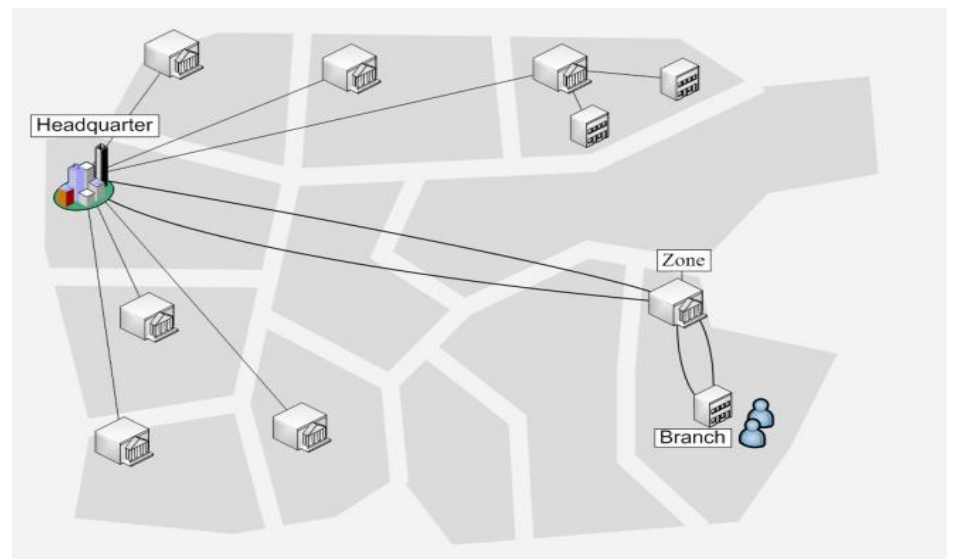

Figure (7).Network topology

Considering network structure, any fault in equipment and communication links between the headquarter and each branch considered as the network components fault, that will lead to undesirable event or the network disconnect. In order to network fault tree analysis, the occurrence of a top event in the network will be shown based on fault in components and communication links. Top event occurring in the fault tree can be divided into two intermediate events through OR logic, on the left side of tree, fault in communications between headquarter and zone center are considered and on the right side faults in communications between zone center and branch are considered. Both of these mentioned communications formed based on parallel links. On the other hand, each time both of these parallel links that are between headquarter and zone center or zone center and branch failed, these mentioned communications are disconnected, in result, fault in each of these communications can be caused by fault in both parallel links and shown by AND logic in fault tree. Finally, fault in each mentioned parallel link can be caused by three basic events (bandwidth usage, packet lost, resource usage), and this information will be obtained by the network protocols. Notice that the network fault tree analysis can be considered in more details but in this paper for ease of expression we omitted those details. In result network fault tree is going to show in Figure (8).

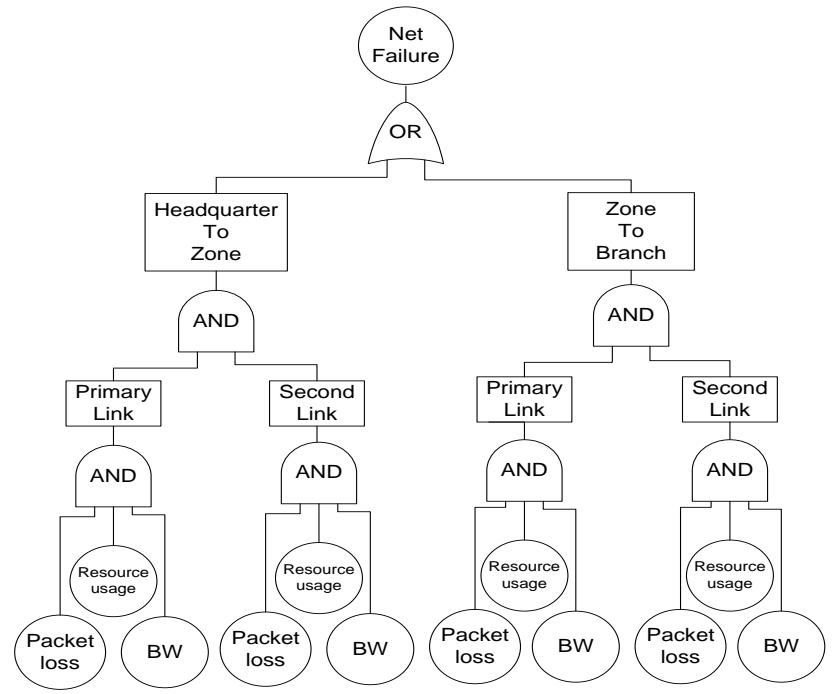

Figure (8). Network fault tree 


\section{Evaluation of the Network Reliability}

Today, the use of fuzzy logic is increasing in systems with indefinite input which are capable of modeling by human knowledge, For example to improve fault tree analysis in such systems with uncertainty in their inputs, it is suggested fault tree analysis to be done by using fuzzy logic. We defined the system and fault tree in the previous section, after that, bottom level of fault tree considered by fuzzy input and membership function between zero and one. Also after considering the bottom level of tree as fuzzy system and apply rules on the fuzzy inputs to find the fuzzy output, then fuzzy output should be converted to de-fuzzy value to use in top level of fault tree.

\section{5-1. Overview}

As mentioned, because basic events are indefinite, fuzzy logic should be used in bottom level of tree. In order to analyses fault tree by fuzzy logic, at first basic events are converted to its fuzzy value by meaning of membership functions. Then apply proposed rules on the fuzzy inputs to find the fuzzy output. After those, results of bottom level of tree are defuzzificated by Larsen/Mirror method and those results are the probability of basic events that can be used in top level of tree to evaluate probability of top event [10]. For a better understanding of words, an example is provided.

In a network, obtained data from SNMP protocols for a communication link and its components are as follows.

Packet loss $=27 \%$, Bandwidth usage $=40 \%$, Resource usage $=50 \%$

Because above data are indefinite, the fuzzy method is used to calculate the fuzzy input value in membership function of Figure (9).

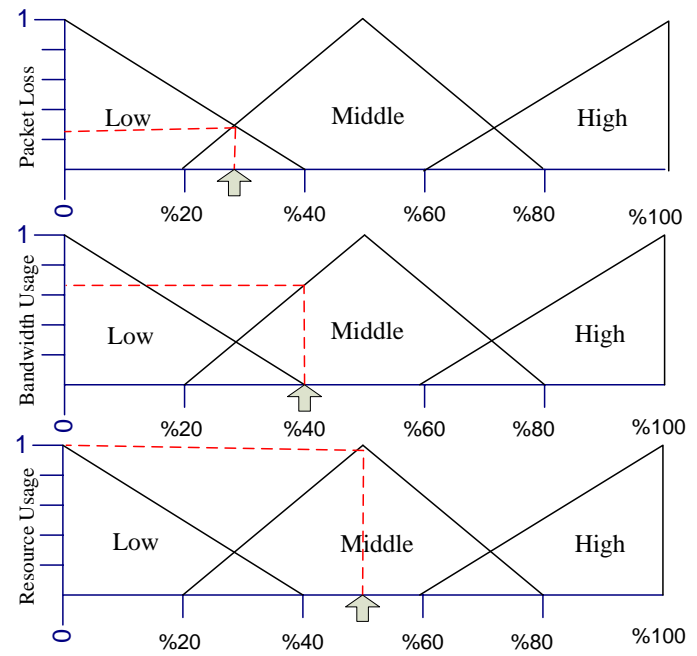

Figure (9). Set point $(\mathrm{P}=\mathbf{2 7 \%}),(\mathrm{B}=40 \%),(\mathrm{R}=50 \%)$

$\mu(x)=\left\{\mu_{\text {Low }}, \mu_{\text {Mid }}, \mu_{\text {High }}\right\}$

Packet loss $=27 \% \rightarrow \mu_{p}(0.27)=\{0,0.25,0.25\} \rightarrow$ Low, Mid 
Bandwidth Usage $=40 \% \rightarrow \mu_{B}(0.4)=\{0,0.62,0\} \rightarrow$ Mid
Resource Usage $=50 \% \rightarrow \mu_{R}(0.5)=\{0,0.5,0\} \rightarrow$ Mid

Mention points in membership function activate two rules in Table (2) and fuzzy output value obtained by fuzzy set theory as follows.

Table (2).Active rules

\begin{tabular}{|c|c|c|c|c|c|c|c|c|c|c|c|c|c|c|c|c|c|c|c|c|c|c|c|c|c|c|c|c|}
\hline \multirow{3}{*}{ 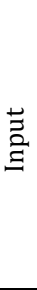 } & n & $\mid \begin{array}{l}3 \\
0 \\
0 \\
-1\end{array}$ & 3. & \begin{tabular}{|l}
3 \\
0 \\
0
\end{tabular} & & 3 & ב్త & 3 & 艿 & 芆 & 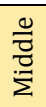 & $\frac{0}{\tilde{z}}$ & $\begin{array}{l}\frac{0}{\tilde{z}} \\
\frac{0}{2} \\
\end{array}$ & 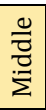 & $\begin{array}{l}\frac{0}{\tilde{z}} \\
\stackrel{0}{\Sigma} \\
\dot{\Sigma}\end{array}$ & $\begin{array}{l}\frac{0}{\tilde{z}} \\
\stackrel{\vec{Z}}{\Sigma}\end{array}$ & $\begin{array}{l}\frac{0}{\tilde{\sigma}} \\
\frac{0}{2} \\
\Sigma\end{array}$ & $\begin{array}{l}\frac{0}{\tilde{\sigma}} \\
\frac{0}{\Sigma} \\
\end{array}$ & $\frac{\mathscr{v}}{\tilde{z}}$ & 节 & $\begin{array}{l}\frac{7}{600} \\
\square 0\end{array}$ & 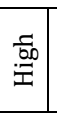 & 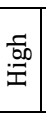 & 㺃 & $\mid \begin{array}{l}\frac{5}{000} \\
.00 \\
.01\end{array}$ & 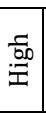 & $\begin{array}{l}\frac{\sigma}{600} \\
.10\end{array}$ & 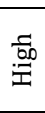 \\
\hline & & $\mid \begin{array}{l}3 \\
0 \\
0\end{array}$ & 3 & \begin{tabular}{|l}
3 \\
3 \\
0
\end{tabular} & & $\frac{0}{\frac{0}{7}}$ & & & $\widetilde{00}$ & & $\begin{array}{l}3 \\
0 \\
9\end{array}$ & $\begin{array}{l}3 \\
0 \\
0\end{array}$ & 3 & & & & 苟 & 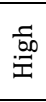 & $\frac{5}{00}$ & 3 & 3 & 3 & & $\begin{array}{l}\frac{0}{\tilde{z}} \\
\stackrel{\vec{z}}{\Sigma}\end{array}$ & \begin{tabular}{|l|}
$\frac{0}{z}$ \\
$\dot{z}$ \\
$\dot{z}$
\end{tabular} & 氜 & $\begin{array}{l}\overrightarrow{5} \\
.000 \\
.7\end{array}$ & \\
\hline & u & \begin{tabular}{|l|}
3 \\
0 \\
0 \\
9
\end{tabular} & $\frac{\stackrel{0}{z}}{\bar{z}}$ & $\stackrel{.50}{.00}$ & 3 & $\frac{0}{7}$ & $\frac{5}{00}$ & 3 & $\begin{array}{l}\stackrel{0}{\frac{\sigma}{\sigma}} \\
\stackrel{0}{\Sigma}\end{array}$ & & $\begin{array}{l}3 \\
0 \\
0\end{array}$ & $\begin{array}{l}\frac{0}{\sigma} \\
\frac{\sigma}{0} \\
\Sigma\end{array}$ & & $\begin{array}{l}3 \\
0 \\
0\end{array}$ & & & $\begin{array}{l}3 \\
0 \\
0 \\
\end{array}$ & & $=$ & 3 & & & & $\Sigma$ & & & & \\
\hline & & $\mid \begin{array}{l}\tilde{N} \\
\sim \\
\sim\end{array}$ & $\overleftarrow{\pi}$ & 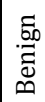 & & . & & $\overline{\vec{d}}$ & $\begin{array}{l}\dot{\Phi} \\
\infty\end{array}$ & $\overrightarrow{\mathbb{b}_{0}}$ & 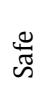 & 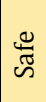 & .0.0 & 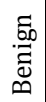 &. & $\dot{\Delta}$ & $\begin{array}{l}\dot{\bar{d}} \\
\text { D. }\end{array}$ & 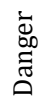 & $\bar{v}$ & .05 & 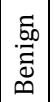 & . & $\dot{\bar{d}}_{\infty}$ & $\overrightarrow{\grave{D}}$ & 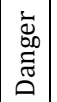 & $\dot{d}$ & $\dot{\vec{d}}$ & à \\
\hline
\end{tabular}

Mid AND Low AND Mid $\rightarrow \max \{0, \min \{0.25,0.62,0.5\})=0.25 \rightarrow$ linkstate $=$ Safe Mid AND Mid AND Mid $\rightarrow \max \{0, \min \{0.25,0.62,0.5\}\}=0.25 \rightarrow$ link state $=$ Benign then $\rightarrow \quad \mu_{\text {Link state }}=\{0.25,0.25,0\}$

After calculating the fuzzy output value, in order to defuzzificating by Larson method we apply $(\mu)$ factor to output membership function, show in Figure (10) [9].

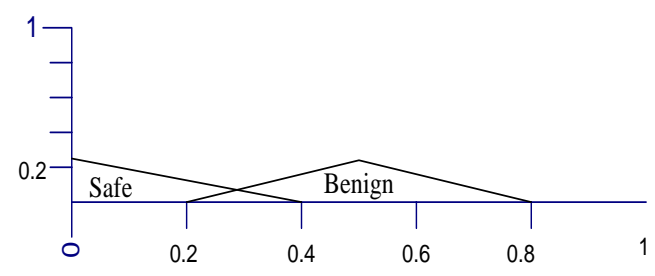

Figure (10).Link State Membership Function

Then, we evaluate crisp value of fuzzy output by using the above output membership function and following equations as a probability of basic event.

$c=$ centriod,$A=$ area $, a=\mu \times A, \frac{\sum a_{i} c_{i}}{\sum a_{i}}=$ Defuzzy $\rightarrow$

probability of basic event $=\frac{0 \times(0.4 \times 0.25)+0.5 \times(0.25 \times 0.3)}{0.1+0.075}=0.214$ 
After finding probability of basic event by above method. We evaluate probability of top level of tree based on basic rules of probability that already described. Equation of top event probability in network fault tree can be expressed as follows.

$$
\begin{aligned}
& p(\text { Net Fail })=p(\text { HQ to Zo })+p(\text { Zo toBr }) \\
& p(\text { HQ to } Z o)=p(\text { pri Link }) \times p(\text { Sec Link }) \\
& p(\text { Zo to } B r)=p(\text { pri Link }) \times P(\text { Sec Link }) \\
& p(\text { pri Link })=p(\text { Bandwiath Usage }) \times p(\text { Resource Usage }) \times p(\text { Packet Loss }) \\
& p(\text { Sec link })=p(\text { Bandwiath Usage }) \times p(\text { Resource Usage }) \times p(\text { Packet Loss })
\end{aligned}
$$

Assuming that basic event probability of all communication links in network fault tree are equaled to 'above probability value obtained(0.214). by placing this non fuzzy values in above Equation, top event probability can be obtained, and after that by subtracting one from top event probability we have network reliability the process described till now is showing by the following equations.

$$
\begin{array}{ll}
p(\text { HQ to } Z \text { O })=(0.2) \times(0.2)=0.04, & p(\text { Zoto Br })=(0.2) \times(0.2)=0.04 \rightarrow \\
p(\text { Net Fail })=(0.04)+(0.04)=0.08 \quad \rightarrow \text { Relaiability }=1-(0.08)=0.92 \rightarrow \text { Network is safe }
\end{array}
$$

\section{Conclusion}

As was expressed, Keeping networks in a reliable mode and evaluating their reliability is an essential task for network managers. In order to evaluate the reliability of network communication we use fault tree model. On the other hand data obtained by the SNMP protocol are indefinite and are used in determining the basic event probability in many cases there is often uncertainty in the estimation of failure probability of network components and communication links, so network communication has been considered as a fuzzy system, and indefinite information such as bandwidth usage, packets lost and the components resource usage are considered as fuzzy inputs in this system, then we use obtained fuzzy values of the basic events, in order to evaluating network reliability by meaning of network fuzzy fault tree.

\section{References}

[1] Barry W. Johnson,"Book: Design and Analysis of Fault-Tolerant Digital system", University of Virginia, Charlottesville

[2] H.Roberts, W.E.Vesely, D.F.Haasl\& F.F.Goldberg, "Fault Tree Handbook", U.S. Nuclear Regulatory Commission Washington

[3] David Hucaby,"Book: CCNP Switch 642-813", Cisco press.

[4] Lotfi A Zadeh."Book: Fuzzy Sets, Fuzzy Logics, and Fuzzy Systems", Univ. of California, Berkeley

[5] E. Stephan, France Telecom, "[RFC 5644, 4148], IP Performance Metrics Registry",University of Surrey

[6] J. Schoenwaelder, "[RFC 4789], Simple Network Management Protocol (SNMP) over IEEE 802 Networks" International University Bremen.

[7] W. Lai, AT\&T Labs, "[RFC 4128], Bandwidth Constraints Models for Differentiated Services" June 2005

[8] G. Almes, S. Kalidindi "[RFC 2680] , One-way Packet Loss Metrics for IPPM"

[9] Timothy J. Ross, "Book: Fuzzy Logic with Engineering Applications", PhD, Stanford University Professor. 